\title{
Europäische Integrationsvorgänge und die räumliche Entwicklung Österreichs - eine prognostische Skizze
}

\section{Fragestellung}

Die wissenschaftliche Befassung mit zukünftigen Entwicklungen ist in der Geographie an sich nicht neu. Viele Untersuchungen befassen sich mit Fragen der Bevölkerungs- und Wirtschaftsentwicklung, die aus gegenwärtigen Erfahrungen auf Zukünftiges zu schließen versuchen. Besonders für Zwecke der Raumplanung wird gefragt, wie sich Regionen als Ganzes voraussichtlich entwickeln werden, um sodann zu überlegen, welche Maßnahmen zum Erreichen bestimmter Ziele notwendig wären. Wenn auch solche «Totalprognosen» die eigentliche Aufgabenstellung einer "prognostischen Geographie» sein sollten (ELSASSER 1976, S. 52), sind die dafür erforderlichen Methoden noch keineswegs ausreichend entwickelt (LICHTENBERGER 1988, S. 369). Da diese Methoden auch von den wissenschaftstheoretischen Positionen der Forschung abhängen, ergeben sich unterschiedliche Anforderungen an die Quantifizierung und Qualifizierung von zukünftig zu erwartenden oder wahrscheinlichen Ereignissen. Weiters stellt sich die Frage, ob Ergebnisse von Prognosen einer ethischen Bewertung unterzogen werden sollen (WEICHHART 1986). Angesichts dieser Problematik darf es einen nicht wundern, daß in vielen Sachfragen immer noch mit (unkontrollierbaren) Vorstellungen über zukünftige Entwicklungen auf der Basis allgemeinen Wissens über räumliche Strukturen und deren Veränderungen das Auslangen gefunden werden muß.

Der folgende Beitrag geht davon aus, daß die Republik Österreich im Juli 1989 offiziell bei den Europäischen Gemeinschaften (EG) um Aufnahme als Vollmitglied angesucht hat. Er versucht skizzenhaft die Auswirkungen dieses politischen Entschlusses auf die räumliche Entwicklung des österreichischen Staatsgebietes zu prognostizieren. Dabei wird sich zeigen, daß wichtige Komponenten der zukünftigen Entwicklung nur zum Teil räumlich differenziert werden können. Auf diese Problematik hat der Autor schon in einer früheren Arbeit hingewiesen (STiglbauer 1985).

Mit dem Beitrittsansuchen Österreichs, das sich ausdrücklich nur auf die Mitgliedschaft bei der Europäischen Wirtschaftsgemeinschaft (EWG), der Europäischen Gemeinschaft für Kohle und Stahl (EGKS) und der Europäischen Atomgemeinschaft
(EAG) bezieht, wird die Teilnahme am «Einheitlichen Europäischen Binnenmarkt» der Europäischen Gemeinschaften angestrebt. In diesem Binnenmarkt, dessen Konzept am 17. Dezember 1985 in den «Einheitlichen Europäischen Akten» festgelegt worden ist, sollen nach dem schon erreichten zollfreien und ungehinderten Warenaustausch innerhalb der EG die folgenden vier wirtschaftlichen Grundfreiheiten: Freizügigkeit der Arbeitnehmer, Niederlassungsfreiheit, Freiheit des Dienstleistungsverkehrs und Freiheit des Zahlungs- und Kapitalverkehrs (bei gemeinsamem Außenzolltarif gegenüber Drittländern) bis 1992 ermöglicht sein (BUNDESMINISTERIUM FÜR AUSWÄRTIGE ANGELEGENHEITEN 1989, S. 167). Da diese politischen Zielsetzungen zu einem weiteren Liberalisierungsschub führen werden und sich daraus Sachzwänge auch für außerhalb der EG liegende Staaten, vor allem in ihrer Wettbewerbsfähigkeit, ergeben, wurde - gedrängt von der Wirtschaft - das Beitrittsansuchen gestellt. In der Tat ist Österreich ja mit dem EGRaum wirtschaftlich besonders intensiv verbunden, wozu das 1972 in Kraft getretene Assoziierungsabkommen zwischen Österreich und den EG für den stufenweisen Abbau der gewerblich-industriellen Zölle und einige Regelungen für den Agraraußenhandel besonders beigetragen haben (vgl. Abschnitt 2). Diesen Vorteil nützen auch die übrigen Staaten der «Europäischen Freihandelsassoziation» (EFTA), die zusammen mit den EG den sog. «Gemeinsamen europäischen Wirtschaftsraum» bilden . Das österreichische Beitrittsansuchen birgt für die EG nicht unbedeutende Probleme wegen der Neutralität Österreichs und wirft Fragen der Stellung der EG innerhalb der politischen Ordnung Europas auf (vgl. Abschnitt 4). Die von den EG getroffene Entscheidung hat wiederum Einfluß auf den Zeitpunkt eines möglichen Beitritts Österreichs in die EG und auch auf die künftige räumliche Entwicklung Österreichs (vgl. Abschnitte 5 und 6).

Karl Stiglbauer, Prof., Dr., Institut für Geographie der Universität Wien, Universitätsstr. 7, A-1010 Wien 


\section{Die wirtschaftliche Verflechtung Österreichs mit Europa}

Die österreichische Volkswirtschaft, bezogen auf einen kleinen europäischen Binnenstaat mit rund $84000 \mathrm{~km}^{2}$ und rund 7,6 Millionen Einwohnern, hat einen vergleichsweise hohen Entwicklungsstand. Das österreichische Bruttonationalprodukt 1987 (pro Kopf und zu Wechselkursen berechnet) lag allerdings weit unter jenem der Schweiz (167; Österreich $=100$ ) sowie der nordischen Staaten (Dänemark und Norwegen: 128, Schweden: 122 und Finnland: 115). Auch der Abstand zur Bundesrepublik Deutschland ist noch groß (118); Frankreich hatte fast den gleichen Pro-Kopf-Wert wie Österreich (101). Die Durchschnittswerte aller übrigen EGStaaten lagen jedoch unter jenem Österreichs: Großbritannien (75), Niederlande (94), Belgien (90), Italien (84), Irland (53), Spanien (48), Griechenland (30) und Portugal (23!) (ÖSTERR. STATISTISCHES ZENTRALAMT 1988, S. 558). Der 11. Platz Österreichs in der internationalen Rangliste des Bruttosozialproduktes (unter Einschluß von Japan, den USA und Kanada) und andere Faktoren wie der Bildungsstand der Bevölkerung zeigen, daß Österreich ein hohes wirtschaftliches Entwicklungspotential in die EG einbringen kann.

Die Statistik des österreichischen Außenhandels und des Fremdenverkehrs belegt anschaulich die hohe Intensität der wirtschaftlichen Verflechtungen mit den EG. Österreich ist damit und hinsichtlich seiner gesamten Gesellschaftsordnung eindeutig «westorientiert» und kein Staat «zwischen» Ost und West (LICHTENBERGER 1988a).

Entsprechend Tabelle 1 entfallen rund zwei Drittel des österreichischen Außenhandels auf den EGRaum. Hauptpartner sind dabei die Bundesrepublik Deutschland und Italien. Der Anteil des EGund EFTA-Raumes zusammen beträgt sogar rund drei Viertel des Wertes des österreichischen Außenhandels. Aus der Sicht der EG ist Österreich (1987), gemessen in Prozenten am EG-Gesamtexportvolumen, nach den USA, der Schweiz und Schweden das viertgrößte Abnehmerland. Danach kommt erst Japan. Noch stärker ist die regionale Abhängigkeit von den EG im Fremdenverkehr. Von den rund 85,69 Mio Ausländerübernachtungen in Österreich (1987) entfielen 89\% auf Touristen aus dem EGRaum; zusammen mit den EFTA-Staaten belief sich dieser Anteil sogar auf 94\%. Bei allen Chancen, die Österreich durch die Verstärkung seiner Ostkontakte in Zukunft auch haben mag: Diese werden die Verflechtungen mit Westeuropa nicht aufwiegen können! Es würde zu weit führen, noch andere wichtige Wirtschaftsverflechtungen nachweisen zu wollen. Bemerkt sei noch, daß Österreich von sich aus seine Währung an die D-Mark gebunden hat und deren Kursänderungen folgt.

\section{Die Frage des Transitverkehrs durch Österreich}

Der Transitverkehr durch Österreich ist im letzten Jahrzehnt so angewachsen, daß die ökologischen Auswirkungen und Beeinträchtigungen der Bevölkerung durch Lärm im Nahbereich der Verkehrswege ein unerträgliches $\mathrm{Maß}$ erreicht haben. Protestbewegungen waren die Folge. Mit 1. Dezember 1989 wird durch Verordnung des zuständigen Bundesministers für Verkehr eine Geschwindigkeitsbeschränkung sowie das Nachtfahrverbot für «laute» Lastkraftwagen (LKW) in Kraft treten. Demzufolge dürfen zwischen 22 Uhr und 5 Uhr auf den Autobahnstrecken Inntal, Brenner, Tauern, Innkreis, Phyrn und Rheintal LKW über 7,5 t Gesamtgewicht nur dann fahren, wenn sie den neuen Lärmschutzbestimmungen gerecht werden. Für besondere Transporte wie für Milch oder Vieh wird es befristete Ausnahmeregelungen geben. Diese angekündigte Maßnahme hat schon zu Straßenblockaden von Frächtern und zu heftigen politischen Reaktionen bei den EG und in der BRD geführt.

Die rapide Zunahme des Straßen-Gütertransitverkehrs durch Österreich von knapp über 5 Mio $t$ im Jahre 1972 auf 21,6 Mio t im Jahre 1988 geht auf mehrere Ursachen zurück: Einer der Hauptgründe ist die gewaltige Steigerung der Transporte im Verkehr nach und von Italien. Dazu kommt die zunehmende Verlagerung der Gütertransporte von der Schiene auf die Straße (so stieg der Bahn-Gütertransitverkehr durch Österreich zwischen 1972 und 1988 nur unwesentlich an und lag 1988 bei 10,1 Mio t); eine dritte Ursache ergibt sich aus der (weisen) Verkehrspolitik der Schweiz, die schon sehr früh eine Beschränkung des Straßengüterverkehrs durch die Festlegung des Höchstgewichts für LKW mit $28 \mathrm{t}$ (gegenüber der Praxis von rund $40 \mathrm{t}$ und darüber) vorgenommen hat und deshalb auf der Straße nur einen Transit von rund 1 Mio t im Jahre 1988 (gegenüber 10,6 Mio t Bahntransit) zu bewältigen hatte. Wegen dieser Beschränkung haben viele Frächter Umwege von oft mehreren hundert Kilometern in Kauf genommen und den Weg über den Brenner in Österreich gewählt.

Eine Lösung muß gefunden werden. Sie sollte erstens über eine funktionell sinnvolle Reduzierung des Verkehrsaufkommens (schon der ökologischen Folgewirkungen wegen) und zweitens durch größtmögliche Verlagerung des Verkehrs auf die Schiene angestrebt werden. Dafür bietet sich der Huckepack-Verkehr oder eine verstärkte Verwendung von Bahn-Containern an. Die zuständigen Bahnverwaltungen haben auf diese Erfordernisse hin viel zu spät reagiert. Nun sind, wie auch in der Schweiz, Projekte für Bahntunnels in Vorbereitung, die allerdings erst Anfang des nächsten Jahrhunderts realisiert werden können, so der geplante rund $55 \mathrm{~km}$ lange Brennertunnel zwischen Innsbruck und Franzensfeste, nördlich von Brixen. 
Tab. 1 Regionale Ausrichtung des österreichischen Außenhandels, gemessen am Wert der Ein- und Ausfuhr (1987) und der Tourismusverflechtung, und diese gemessen an der Zahl der Ausländerübernachtungen (Kalenderjahr 1987) in Prozent

\begin{tabular}{|c|c|c|c|c|c|c|c|}
\hline & & Einfuhr & & Ausfuhr & \multicolumn{3}{|c|}{ Übernachtungen } \\
\hline \multicolumn{2}{|l|}{$E G$} & 68,00 & & 63,40 & & 89,37 & \\
\hline davon: & $\begin{array}{l}\text { BRD } \\
\text { Italien } \\
\text { Frankreich } \\
\text { Benelux }\end{array}$ & & $\begin{array}{r}44,20 \\
9,40 \\
4,10 \\
5,20\end{array}$ & & $\begin{array}{r}34,80 \\
10,40 \\
4,50 \\
5,00\end{array}$ & & $\begin{array}{r}64,11 \\
1,97 \\
3,11 \\
13,51\end{array}$ \\
\hline \multicolumn{2}{|l|}{ EFTA } & 7,80 & & 11,10 & & 4,88 & \\
\hline davon: & Schweiz & & 4,70 & & 7,40 & & 2,61 \\
\hline \multicolumn{2}{|c|}{ Osteuropa } & 6,80 & & 9,00 & & 1,34 & \\
\hline davon: & $\begin{array}{l}\text { UdSSR } \\
\text { Ungarn } \\
\text { CSSR } \\
\text { Polen } \\
\text { Jugoslawien }\end{array}$ & & $\begin{array}{l}2,10 \\
1,50 \\
1,40 \\
1,00 \\
1,00\end{array}$ & & $\begin{array}{l}2,50 \\
1,90 \\
1,10 \\
0,90 \\
2,00\end{array}$ & & $\begin{array}{l}0,05 \\
0,58 \\
0,12 \\
0,09 \\
0,43\end{array}$ \\
\hline \multicolumn{2}{|c|}{$\begin{array}{l}\text { übriges Europa } \\
\text { Rest derWelt } \\
\text { insgesamt }\end{array}$} & $\begin{array}{r}1,00 \\
16,40 \\
100,00\end{array}$ & & $\begin{array}{r}2,10 \\
14,40 \\
100,00\end{array}$ & & $\begin{array}{r}4,41 \\
100,00\end{array}$ & \\
\hline
\end{tabular}

Quelle: Statistisches Jahrbuch der Republik Österreich, 1988, Wien (S. 375, 389 und 395).

\section{Der Neutralitätsstatus Österreichs und die politische Ordnung in Europa}

Während die organisatorischen Vorarbeiten zur Einrichtung des Europäischen Binnenmarktes bei den Zentralen der EG in Brüssel und teils auch bei den Mitgliedstaaten auf vollen Touren laufen, werden keine entscheidenden Verhandlungen über den Beitritt Österreichs erfolgen. Daher wird es also eine Wartezeit von mehreren Jahren geben. Unter Einrechnung der erforderlichen Übergangsfristen käme der Binnenmarkt für Österreich somit erst um die Jahrtausendwende zum Tragen. Für den EGBeitritt ist auch das noch ungelöste Problem des österreichischen Neutralitätsstatus zu berücksichtigen.

Nach dem Zweiten Weltkrieg blieb Österreich bis 1955 besetzt. Der mit den USA, der Sowjetunion, Großbritannien und Frankreich unterzeichnete «Staatsvertrag» gab Österreich sodann die volle Souveränität zurück, u. a. mit der Auflage, «keine wie immer geartete politische oder wirtschaftliche Vereinigung mit Deutschland» einzugehen (Art. 4 Abs. 1). Die Vertragspartner verpflichteten sich ihrerseits, für die Einhaltung dieser Bestimmung besonders zu sorgen. Aufgrund dieser Klausel hat die Sowjetunion seitdem jeden Versuch einer EG-Annäherung Österreichs unter Hinweis auf diese Vertragsverpflichtung beurteilt und den Verhandlungs- spielraum mit den EG stark eingeschränkt. Dies könnte auch in Zukunft so sein. Seitens der EG schafft die von Österreich eingegangene Selbstbindung als neutraler Staat («nach Schweizer Muster»), ebenfalls 1955 beschlossen, Probleme, weil sich die Neutralität mit den Zielen einer integrierten politischen Unior, die ja schon durch den «Vertrag über die Europäische Zusammenarbeit auf dem Gebiet der Außenpolitik» (1985) ein institutionelles Fundament erhalten hat, nicht einfach vereinbaren läßt. Österreich wiederum weist darauf hin, daß seine Neutralität doch als ein Beitrag zu Frieden und Sicherheit innerhalb Europas gewertet werden sollte.

Die Neutralität Österreichs ist nunmehr auch vor dem Hintergrund der stürmisch verlaufenden Entwicklungen in dem einst so mächtigen Ostblock Europas zu sehen. Wie wird die politische Landkarte Europas von morgen aussehen? Die Diskussion dieser Frage muß in geopolitischen Dimensionen geführt werden. Nach der jahrzehntelangen Konfrontation der beiden Supermächte, die gigantische Beträge des Volkseinkommens in ihre Verteidigungssysteme investierten und die Europa in die Militärblöcke der Nordatlantischen Allianz (NATO) und der Staaten des Warschauer Verteidigungspaktes (WVO) teilten, ist nun eine beiderseitige Abrüstung im Gange. Dazu kommen noch die nicht übersehbaren Auswirkungen der von Michael GORBA- 
TSCHOW in der Sowjetunion ausgelösten Reformbewegungen, die in Polen, Ungarn und in der Deutschen Demokratischen Republik, sowie mit ersten Anzeichen auch in Bulgarien, zu großen Umwälzungen, teils schon zum Machtverlust der Kommunistischen Parteien geführt haben, und auch in der Sowjetunion eine große Instabilität auslösen.

Schon werden Szenarien diskutiert, wie sich die neue politische Ordnung Europas einstellen könnte (vgl. hiezu auch HaLl, 1977). Von GORBATSCHOW stammt die Vorstellung des «gemeinsamen Hauses Europas», eines Wirtschaftsraumes aus den EG, der EFTA und dem RGW. Der emigrierte russische Historiker Michail vOSLENSKY geht von einem Zerfall des russischen «Kolonialreiches» aus: die baltischen Staaten werden frei, ebenso die Ukraine und die Moldawische Sowjet-Republik. Die baltischen Staaten würden mit Polen, der Tschechoslowakei und Ungarn ein neues neutrales Mitteleuropa bilden und so den russischen Sicherheitsbedürfnissen entgegenkommen. Der britische Journalist Robert COTTRELL hat dieses Szenario weiter entwickelt und besonders die Relationen zu den EG berücksichtigt. Er geht zunächst davon aus, daß Österreich, Norwegen, Schweden und Finnland in die EG aufgenommen werden (nicht aber die Schweiz). Eine Wiedervereinigung der beiden deutschen Staaten wäre in nächster Zeit nicht zu erwarten. Wohl würden sich aber beide Staaten ebenfalls neutral einstellen, was im übrigen große strategische Probleme aufwerfen würde: Die BRD müßte in einem solchen Falle aus der NATO ausscheiden, und die DDR müßte ihre bisher wichtige strategische Funktion als Vorposten der Sowjetunion aufgeben, ganz abgesehen von der historisch berechtigten Befürchtung der Wiedererstarkung eines sich vereinenden nationalen Deutschlands. COTTRELL nimmt an, daß sich die EG um die Neuordnung Europas aktiv mit neuen Formen einer Mitgliedschaft oder mittels besonderer Assoziierungen zur Gewährleistung enger wirtschaftlicher Zusammenarbeit bemühen würden, wodurch der militärisch neutrale Korridor des östlichen Mitteleuropas wirtschaftlich auf das engste mit Westeuropa verbunden wäre (SPERL 1989). Westeuropa erblickt im östlichen Europa einen groBen Absatzmarkt. Rechnet man die Bevölkerung der EG (Mitgliedsstaaten), der EFTA und der RGW-Staaten in Europa (einschließlich der Sowjetunion) zusammen, so kommt man auf ein Marktpotential von 749 Mio Einwohner, wovon auf die EG rund 323 Mio Einwohner entfallen. So gesehen, hätte das «gemeinsame Haus» Europas auch eine solide ökonomische Untermauerung.

Noch ist es ganz ungewiß, ob solche Szenarien, wenn überhaupt, Realität gewinnen werden. Für Österreich erweist sich aber die zukünftige Gestaltung seines derzeit an sich schon guten Verhältnisses zu den «östlichen» Nachbarstaaten, die sich ja zuvor jahrzehntelang durch Stacheldrahtverhaue und Mi- nengürtel abgeschottet hatten, als von großer Tragweite. Wird Österreich mit seinem international weit ausstrahlenden Zentrum Wien entweder als neutraler Staat wie bisher um gute nachbarliche Kontakte bemüht sein müssen, oder wird es solche Funktionen als ein Mitglied der EG wahrzunehmen haben? Wie hätte vor allem Wien zu reagieren, wenn sich Budapest als neues «Einfallstor» in das neue neutrale Mitteleuropa etablieren möchte? Wenn auch keine eindeutige Antwort auf diese Fragen gegeben werden kann, sind in jedem Falle raumbedeutsame Auswirkungen zu erwarten.

\section{Regionale Auswirkungen der Integration auf die österreichische Wirtschaft}

Vor dem Beitrittsansuchen Österreichs wurden vor allem im Auftrage der Bundesregierung zahlreiche Gutachten ausgearbeitet, so über die Frage der Neutralität (HUMMER und SCHWEITZER 1987) und über viele Fragen der Wirtschaft (u. a. BREUSS und SCHEBEK 1989; BREUSS, HANDLER Und STANKOVSKY 1989; SCHNEIDER 1988; szOPO 1988). Des weiteren äußerten sich die Interessenvertretungen, unterstützt von einigen Tageszeitungen. Auch fanden verschiedene Vortragsveranstaltungen statt, deren Ergebnisse publiziert wurden (GLATZ und MOSER 1989; MOSER 1988; SCHERB und MORAWETZ 1988 u. a.).

Der Wissensstand über die teils sehr verzweigten Probleme eines Einbezugs der österreichischen Wirtschaft in den EG-Binnenmarkt konnte so wesentlich ausgeweitet werden. Es würde aber den Rahmen dieses Beitrages sprengen, auf die vielen Untersuchungsergebnisse und ihre Bewertung hier einzugehen. Da zumeist in globalen Kategorien diskutiert wurde, geriet die Behandlung der räumlich differenzierten Integrationsprobleme in den Hintergrund. Dennoch liegen auch dazu einige Aussagen vor: Die ersten größeren Studien über die regionalen EG-Auswirkungen stammen von w. JÄGER (1989) und R. WURZER (1970). Neuere Untersuchungen lieferten insbesondere K. STIGLBAUER (1985). E. LICHTENBERGER (1988; 1989: siehe Literaturverzeichnis, Abschnitt 7), die ÖSTERREICHISCHE RAUMORDNUNGSKONFERENZ (1989; siehe Mitarbeiterverzeichnis) und TÖDTLING-SCHÖNHOFER (1989).

Die österreichische Industrie (einschließlich des produzierenden Gewerbes), deren regionaler Schwerpunkt im östlichen Staatsgebiet liegt, ist be reits seit vielen Jahren dem Konkurrenzdruck der EG ausgesetzt. Ein Eintritt in den EG-Binnenmarkt würde diese Konkurrenz zwar erhöhen, dafür aber anderweitig günstige Voraussetzungen schaffen - so würden beispielsweise notwendige und teils hinausgeschobene Strukturverbesserungen erzwungen werden. Der Anpassungsdruck würde die Betriebe der verstaatlichten Industrie treffen und die Probleme des «alten Industriegebietes» in der Ober- 
steiermark verschärfen. Die privaten Betriebe haben sich schon in letzter Zeit besonders investitionsfreudig erwiesen, wenngleich der Anteil an Produkten des High-Tech-Sektors noch immer zu niedrig ist und viele Grundstoff- und Halbfertigwarenerzeugungen vorherrschen. So ist zum Beispiel die Autozulieferindustrie in Österreich stark vertreten. Zu berücksichtigen ist auch, daß Österreich im Anteil der überwiegend im ausländischen Eigentum stehenden Unternehmen (voran die BRD, Schweiz und Japan), bezogen auf die Beschäftigten, im Spitzenfeld der OECD-Länder liegt. Leider besitzen diese Unternehmen nur eine geringe Verflechtung mit der übrigen Industrie Österreichs (vgl. PICHL 1989). Nach G. PALME (1989; ÖSTERR. RAUMORDNUNGSKONFERENZ 1989), der die Regionalstruktur der Industrie Österreichs im Hinblick auf ihre Zuordnung zu Produktionszyklen untersuchte, werden nur die Regionen mit vielen Arbeitsplätzen im Verarbeitungs- und Technologiesektor, zumeist im ländlichen Raum gelegen und wenig gewichtig, sowie die derzeit wenig industrialisierten und entwicklungsschwachen peripheren Regionen mit jüngeren Industrieansiedlungen bei einem EG-Beitritt überdurchschnittliche Chancen erhalten. Für alle übrigen Regionen wären nur durchschnittliche Chancen anzunehmen. Von überdurchschnittlichen Risiken betroffen werden nur die Agglomerationen sein.

$\mathrm{Zu}$ bedenken sind auch die regionalen Unterschiede im Arbeitsmarkt. Während im Osten Österreichs als Folge der Überalterung ein anhaltender Arbeitskräftemangel besteht (weshalb eine Zuwanderung aus den benachbarten Oststaaten als Vorteil gewertet wird), kommt es im Westen Österreichs durch (zwar rückläufige) Geburtenüberschüsse zu einem anhaltenden Bedarf an Arbeitsplätzen (FASSMANN; SAUBERER, in: LICHTENBERGER 1989). Da jetzt schon in Süddeutschland (mit München als Zentrum) Arbeitsmärkte mit einem höheren Lohnniveau als im westlichen Österreich bestehen, würde unter den Bedingungen des Binnenmarktes die Abwanderung oder ein verstärktes Weitpendeln gefördert werden. Der Fremdenverkehr in Österreich besitzt bereits einen sehr hohen Ausbaugrad. In vielen Gebieten zeigen sich infolge der anhaltenden Konzentration verschiedene Überlastungserscheinungen, so daß z. B. Seilbahnausbauten schon gestoppt werden. Von großer Tragweite ist die Frage, ob beim EGBinnenmarkt die derzeit zumeist von Ortsansässigen geführten Klein- und Mittelbetriebe des Hotelund Gaststättensektors durch Einströmen von ausländischem Kapital (Errichtung von Großhotels, auch von Appartementhäusern und Zweitwohnungen und zur Erschließung neuer Gebiete) verdrängt werden würden. Nach ZIMMERMANN (in: LICHTENBERGER 1989) müßte mit dem Aufkommen eines solchen fremdgesteuerten Tourismus besonders in den imageträchtigen Standorten gerechnet werden (vor allem in den Städten). Zur Steuerung solcher Pro- zesse können aber auch EG-konforme Instrumente der Raumordnung und der Grundverkehrspolitik eingesetzt werden, weshalb ein neuer Handlungsbedarf entstehen wird (WEBER, in: ÖSTERR. RAUMORDNUNGSKONFERENZ 1989).

Die österreichische Landwirtschaft würde zu den «Hauptverlierern» eines EG-Beitritts zählen, weil sie derzeit der europäischen Konkurrenz nur zum Teil gewachsen ist. Die vorwiegend «ökosozial» ausgerichtete österreichische Agrarpolitik würde durch die auf Entwicklung leistungsfähiger Strukturen abgestellte EG-Agrarpolitik ersetzt werden müssen und neue Peripherien schaffen. Preiseinbußen würden sich bei Getreide, Gemüse, Zuchtrindern, Schweinen und bei Eiern ergeben; davon wären sowohl Flachland als auch Berglandgebiete betroffen (SCHNEIDER 1988). Da von der bäuerlichen Landwirtschaft, besonders in den Alpen, auch wichtige Funktionen einer Landschaftspflege geleistet werden (stiglbauer 1985), müßte - wie von den österreichischen Landwirtschaftsvertretern gefordert die EG-Agrarpolitik entsprechend modifiziert werden.

Faßt man diese sektoralen Ergebnisse zusammen, so ergibt sich ein sehr differenziertes Bild. Alle EGExperten sind der Auffassung, daß die «positiven» Effekte des Binnenmarktes zur verstärkten Konkurrenz unter den Regionen führen werden. Einige Regionen würden davon besonders profitieren, andere wieder in der Entwicklung zurückbleiben, was die regionalen Disparitäten vergrößern würde. Bei dieser Problematik sollte man Österreich auch mit den Regionen Europas vergleichen. Schon Stein ROKKAN (1980) hat auf die großen, historisch beständigen Unterschiede zwischen West-, Mittel- und Osteuropa hingewiesen und dabei die Rheinachse als ein besonders gliederndes Element erkannt. Auch nachfolgende Untersuchungen führten zur Abgrenzung einer Zone, die von London über Frankfurt nach Mailand reicht und die höchste Konzentration an Wirtschaftspotential und Innovationskraft besitzt. Sie wurde wegen ihrer geschwungenen Form salopp als «blue banana» bezeichnet. Österreich liegt zwar relativ weit davon ab, gehört aber noch zum Bereich der leistungsstarken Volkswirtschaften und nicht zur Peripherie Europas. Auf die besondere internationale zentralörtliche Bedeutung von Wien im Rahmen der politischen Neuordnung Europas wurde bereits oben hingewiesen. Nach übereinstimmender Meinung der Experten würde der Wiener Raum auch bei einem EG-Beitritt Österreichs zweifelsohne am stärksten profitieren. Günstige Entwicklungschancen besitzt auch der Westen Österreichs, und zwar wegen der räumlichen Nähe zum dynamischen Süden der BRD und wegen seiner schon jetzt stark exportorientierten Industrie. Der Südosten Österreichs weist die relativ größten regionalwirtschaftlichen Probleme auf (TÖDTLINGSCHÖNHOFER, 1989b, S. 152 ff.). Bei einem EG-Bei- 
tritt und bei stärker genützten Standortvorteilen gegenüber dem oberitalienischen und nordjugoslawischen Raum, und auch in bezug auf West-Ungarn, könnten zusätzliche Entwicklungsimpulse erwachsen. Interessant ist das Ergebnis einer Prüfung der Wirtschaftskraft österreichischer Regionen nach den Maßstäben einer Förderungswürdigkeit durch den EG-Regionalfonds (Bruttoregionalprodukt/je Kopf und Arbeitslosenquote): Österreich würde bei einem EG-Beitritt nur in sehr geringem Umfange von den Förderungsinstrumenten des Fonds profitieren, weil die Bundesländer, ausgenommen das Burgenland, nicht unter die vorgegebenen Grenzwerte fallen würden (TÖDTLING-SCHÖNHOFER, 1989a, S. 163 ff.).

\section{Zusammenfassung}

Die Analyse einschlägiger Literatur zeigte eine umfangreiche raumbedeutsame Problematik auf, die mit einem Beitritt Österreichs zur EG aufkommen würde. $\mathrm{Zu}$ erwarten ist ein neuer, besonders qualitativer Wachstumsschub in der regionalen Entwicklung, der allerdings den Einsatz von wirksamen Instrumenten der Regionalpolitik und Raumordnung erforderlich machen wird. Diese Zukunftsvorstellung besitzt jedoch eine größere Unbekannte in der politischen Ordnung Europas im nächsten Jahrzehnt.

\section{Literaturverzeichnis}

BREUSS, F. (1988): Abschätzung möglicher Wohlfahrtseffekte eines österreichischen EG-Beitritts. In: GLATZ, H. und MOSER, H. (Hrsg.) (1989): Herausforderung EG-Binnenmarkt. Kopfüber in die EG? Service-Fachverlag an der Wirtschaftsuniversität Wien, S. 77-108, Wien.

BREUSS, F., HANDLER, H. und STANKOVSKY, J. (1988): Österreichische Optionen einer EG-Annäherung und ihre Folgen. Institut für Wirtschaftsforschung, Wien.

BREUSS, F. und SCHEBECK, F. (1989): Die Vollendung des EG-Binnenmarktes. Gesamtwirtschaftliche Auswirkungen auf Österreich. Makroökonomische Modellsimulationen. Österreichisches Institut für Wirtschaftsforschung, Wien.

BUNDESKANZLERAMT/BUNDESMINISTERIUM FÜR AUSWÄRTIGE ANGELEGENHEITEN (1989): Vortrag an den Ministerrat, betr. Europäische Gemeinschaften (Manus), Wien.

BUNDESMINISTER FÜR AUSWÄRTIGE ANGELEGENHEITEN (1989): Jahrbuch der österreichischen Außenpolitik. AuBenpolitischer Bericht 1988. Kommissionsverlag MANZsche Verlags- und Universitätsbuchhandlung, Wien.
BUNDESREGIERUNG (1989): Bericht der Bundesregierung an den Nationalrat und den Bundesrat über die zukünftige Gestaltung der Beziehungen Österreichs zu den Europäischen Gemeinschaften (Manus), Wien.

ELSASSER, H. (1976): Gedanken zur prognostischen Geographie. In: Geographica Helvetica (1), 49-55, Zürich.

GLATZ, H. und MOSER, H. (Hrsg.) (1989): Herausforderung EG-Binnenmarkt. Kopfüber in die EG? Service-Fachverlag an der Wirtschaftsuniversität Wien, Wien.

HALL, P. (1977): Europa 2000. Duckworth, London.

HANIKA, A. (1989): Bevölkerungsvorausschätzung 1989-2015 des österreichischen Statistischen Zentralamtes für Österreich und die Bundesländer sowie Modellrechnung bis 2050. In: Statist. Nachrichten (N.F.) 44. Jg./8, S. 552-561, Wien

HELLWIG, Ch. (1989): Grenzüberschreitender Güterverkehr in Österreich im Jahre 1987. In: Statistische Nachrichten (N. F.) 44. Jg., H. 4, S. 323-325, Wien.

HUMMER, W. und SCHWEITZER, M. (1987): Österreich und die EWG. Neutralitätsrechtliche Beurteilung der Möglichkeiten der Dynamisierung des Verhältnisses zur EWG. Signum Verlag, Wien.

JÄGER, W. (1988): Vorträge und Aufsätze 1949-1988. Hrsg. Magistratsdirektion Wien, Wien.

LICHTENBERGER, E. (1988a): Österreich - der Staat zwischen West und Ost. In: Geogr. Rundschau 40, Nr. 10, S. $6-12$, Braunschweig.

LICHTENBERGER, E. (1988b): Szenarien der West-Ost-Entwicklung in Österreich: Ein Beitrag der Geographie zur Zukunftsforschung. Einführung. In: 46. Deutscher Geographentag München, 12. bis 16. Oktober 1987, Tagungsbericht und wissenschaftliche Abhandlungen, S. 369-372. Franz Steiner Verlag Wiesbaden $\mathrm{GmbH}$, Stuttgart.

LICHTENBERGER, E. (Hrsg.) (1989): Österreich zu Beginn des 3. Jahrtausends. Raum und Gesellschaft. Prognosen, Modellrechnungen und Szenarien. In: Beiträge zur Stadtund Regionalforschung, Band 9, Österr. Akademie der Wissenschaften, Wien. Darin:

- BAUMHACKL, H.: Szenarien und Modellrechnungen zur Entwicklung des Zweitwohnungswesens in Österreich bis zum Jahr 2011, S. 203-235.

- FASSMANN, H.: Die Zukunft des österreichischen Arbeitsmarkts: Modellrechnungen zur Angebotsentwicklung und zum sektoralen Strukturwandel auf dem Arbeitsmarkt bis 2011, S. 119-145.

- LICHTENBERGER, E.: Gegenwart und Zukunft von Raum und Gesellschaft, S. 15-67;

- dito: Optionen für Europa, S. 237-263;

- MEUSBURGER, P.: Die Effekte des Geburtenrückgangs: Prognosen und Modellrechnungen zur Entwicklung des österreichischen Volksschulwesens bis 2011, S. 101-118;

- PENZ, H.: Die Zukunft der österreichischen Landwirtschaft in der Phase der Überproduktion: Prognosen und Szenarien bis zum Jahr 2000, S. 147-175;

- SAUBERER, M.: Prognosemodell und Szenarien zur räumlichen Bevölkerungsentwicklungsentwicklung Österreichs bis 2030, S. 74-99;

- ZIMMERMANN, F.: Ende des Wachstums und Umbau des Fremdenverkehrs: Szenarien und Modellrechnungen zum österreichischen Fremdenverkehr im Jahre 2000 , S. $177-202$

MOSER, B. (Hrsg.) (1988): EUROPA-Kongreß: Unser Weg in die Europäische Gemeinschaft, Schriftenreihe "Standpunkte", Politische Akademie der ÖVP, Bd. 13, Wien. 
ÖSTERREICHISCHE

RAUMORDNUNGSKONFERENZ

(1989): EG-Integration - Auswirkung auf Raumordnung und Regionalpolitik. Enquete am 14. Juni 1989. In: Schriftenreihe der ÖROK (in Druck) mit Beiträgen von:

- GATTERBAUER, H.: Auswirkungen der EG-Integration auf Bodenordnung und Ausländergrundverkehr;

- KANIAK, J. und KEIL, D.: Auswirkungen der Integrationsbestrebungen auf die regionale Wirtschaftsstruktur und den Handlungsbedarf in der Wirtschaftsförderung;

- PALME, G.: EG-Integration - Auswirkungen auf die Regionalstruktur der Industrie;

- STRAUBE, M.: Auswirkungen der EG-Integration auf Bodennutzung und Siedlungsentwicklung - gesellschaftsrechtlicher Handlungsbedarf;

- WEBER, G.: EG-Integration - Raumordnung im Zugzwang?

ÖSTERREICHISCHES STATISTISCHES ZENTRALAMT (1988): Statistisches Handbuch für die Republik Österreich, Wien.

PALME, G. (1989): Entwicklungsstand der Industrieregionen Österreichs. In: Monatsberichte des Österr. Instituts für Wirtschaftsforschung, Nr. 5, S. 331-345, Wien.

PICHL, C. (1989): Internationale Investitionen. Verflechtung der österreichischen Wirtschaft. In: Monatsberichte des Österr. Instituts f. Wirtschaftsforschung, Nr. 3, S. 161-175, Wien.

ROKKAN, S. (1980): Territories, centres and peripheries: Toward a geoethnic-geoeconomic-geopolitical model of differentiation within Western Europe. In: GOTTMANN, J. (Ed.): Centre and periphery. Spatial variation in politics, Beverly Hills, Page, 163-204.

SZOPO, P. (1988): Folgen einer EG-Annäherung für Wirtschaftsförderung und Wettbewerbspolitik in Österreich. Institut für Wirtschaftsforschung, Wien.
SCHERB, M. und MORAWETZ, I. (Hrsg.) (1988): Der unheimliche Anschluß. Österreich und die EG. In: Österreichische Texte zur Gesellschaftskritik, 35, Verein für Gesellschaftskritik, Wien.

SCHNEIDER, M. (1988): Europäische Integration: Eine schwere Herausforderung für die österreichischen Bauern. Vortrag. In: WIPO-Vorträge 41, Österr. Institut für Wirtschaftsforschung, Wien.

SPERL, G. (1989): Neutraler Korridor von Nord bis Süd? In: Der Standard, 6. November 1989, S. 4, Wien.

STIGLBAUER, K. (1985): Wirkungen der Europäischen Gemeinschaften auf die räumliche Entwicklung Österreichs. In: Ansätze zu einer europäischen Raumordnung, Veröffentl. d. Akademie für Raumforschung und Landesplanung, Band 155, S. 323-364, Curt R. Vincentz Verlag, Hannover.

TÖDTLING-SCHÖNHOFER, H. (unter Mitarbeit von BAROUD, R. und PLITZKA-RICHTER, U.) (1989a): Auswirkungen eines EG-Beitritts Österreichs auf die Instrumente der regionalen Wirtschaftsförderung. In: Schriften zur Regionalpolitik und Raumplanung, 16, hrsg. Bundeskanzleramt, Abt. IV/ 4 - Raumplanung und Regionalpolitik, Wien.

TÖDTLING-SCHÖNHOFER, H. (1989b): Auswirkungen eines EG-Beitrittes Österreichs auf die regionale Wirtschaftsentwicklung und die Regionalpolitik. In: GLATZ, H., MOSER, H. (Hrsg.) (1989): Herausforderung EG-Binnenmarkt. Kopfüber in die EG? Service-Fachverlag an der Wirtschaftsuniversität Wien, S. 135-163, Wien.

WEICHHART, P. (1986): Ethische Probleme und Fragen der Verantwortung in der Geographie. In: Mitteilungen der Österr. Geogr. Gesellschaft, 128, S. 5-33.

WURZER, R. (1970): Expertengutachten. In: Raumordnung für Österreich. Leitlinien und Aktionsprogramm der Bundesregierung - Expertengutachten. Hrsg. Bundespressedienst, Wien. 